\title{
METODOLOGIA BIM E INTEROPERABILIDADE
}

\author{
Alcínia Zita Sampaio \\ Universidade de Lisboa, Instituto Superior Técnico, Dep. Engenharia Civil e Arquitectura \\ Av. Rovisco Pais, Lisboa, Portugal
}

\begin{abstract}
RESUMO
A metodologia Building Information Modelling (BIM) apoia a realização do trabalho colaborativo, baseado na centralização de toda a informação num modelo único e no elevado nível de interoperabilidade das plataformas BIM disponíveis. O presente estudo analisa o processo de transposição de modelos de estruturas entre os sistemas de modelação e de dimensionamento, por recurso a plataformas BIM. São analisadas diversas situações envolvendo as ferramentas de modelação ArchiCAD, Revit e AECOsim e de dimensionamento estrutural SAP, Robot e ETABS. A transposição de informação entre fases é apoiada no formato nativo, quando as aplicações pertencem à mesma casa de software, ou por recurso ao padrão universal de transferência de dados, o formato Industry Foundation Classes (IFC). A análise da capacidade de interoperabilidade verificada em cada fase de transposição de modelos, é avaliada sobre vários casos de estudo, com distinta volumetria e utilização. As conclusões do trabalho permitem reconhecer que a implementação do BIM no projecto de estruturas é positiva, pois proporciona a concentração de informação, a capacidade de avaliar inconsistências na sobreposição de especialidades, e a transferência de resultados entre etapas. Contudo, o nível de maturidade do BIM num gabinete de projecto de estruturas é, ainda, relativamente baixo quando comparado com outros sectores, pois há algum desconhecimento das reais potencialidades do BIM e como se deve actuar perante as inconsistências verificadas. O estudo demostra que, apesar das limitações identificadas ao longo do desenvolvimento dos casos de estudo, causadas essencialmente por problemas de interoperabilidade, a metodologia apresenta vantagens claras no desenvolvimento do projecto.
\end{abstract}

\section{PALAVRAS-CHAVE}

BIM, Projecto de Estruturas, Interoperabilidade

\section{INTRODUÇÃO}

A evolução tecnológica aplicada ao sector da Construção, tem vindo a introduzir potencialidades na agilização da elaboração do projecto, no acompanhamento do processo de construção e, posteriormente, na gestão do edifício. Na década de 60 do século XX, surgiram as aplicações computacionais Computer-Aided Design (CAD), levando o desenho do estirador para o computador, facilitando a execução da documentação gráfica do projecto. No entanto, apesar do apoio reconhecido dos sistemas CAD, estes não garantem ainda uma completa eficiência, na coerência entre as diferentes peças desenhadas de um edifício. Apesar dos avanços da tecnologia dirigidos à representação gráfica, o seu princípio de funcionamento baseia-se na manipulação de entidades gráficas, carecendo da capacidade de arquivar e gerir a informação referente a propriedades físicas dos materiais no edifício. Adicionalmente, a intrínseca colaboração entre os vários técnicos envolvidos no desenvolvimento do projeto, requer uma ágil interação de comunicação e de transmissão de dados entre fases (Sampaio, 2017).

A ideia conceptual da criação de um modelo central, para o desenvolvimento de todo o projecto foi inicialmente mencionado por Eastman (Sacks et al. ,2018), na década de 70 do séc. XX, tendo o termo Building Information Modelling (BIM), começado a ser aplicado, em 1987, quando do lançamento do primeiro produto comercial BIM, o ArchiCAD. A metodologia BIM admite a capacidade de visualização do modelo tridimensional (3D) representativo do edifício, e a centralização de toda a informação associada ao seu projeto. O projeto BIM é elaborado numa base de colaboração entre todos os intervenientes da conceção de uma obra, e ao longo de todo o seu período de vida apoiando uma adequada análise da compatibilização entre os diferentes projetos de especialidade. 
Apesar dos benefícios que têm vindo a ser reconhecidos em relação à implementação do BIM no sector, a sua adopção ao nível da empresa e do gabinete de projectos, impõe desafios organizacionais significativos referentes à adaptação cultural interna de transferência de dados entre fases e de comunicação com parceiros e, ainda, a um investimento inicial significativo em tecnologia e formação (NIBS, 2013). Embora a tecnologia disponível de suporte ao BIM admita, uma abordagem bastante integrada da representação digital de todas as disciplinas do edifício e dos métodos que suportam o crescimento do modelo, durante todas as fases do seu ciclo-de-vida, ainda, há lacunas que a investigação se propõe tentar ultrapassar. A pesquisa aponta em duas direções principais: os fabricantes de software têm vindo a admitir uma incremental capacidade de funcionalidades integradas, referidas como extensions, add-in e plug-in, baseadas no formato nativo do sistema utilizado; a organização internacional BuildingSMART, que agrega empresas de diversos países e que é responsável pela emissão do padrão universal de transferência de dados, o formato Industry Foundation Classes (IFC) (BuildingSMART, 2019), tem vindo a apresentar versões do padrão sucessivamente mais eficientes.

Contudo, o projecto de estruturas, elaborado sobre plataformas de base BIM, requer que o utilizador reconheça as limitações ao nível da interoperabilidade dos sistemas disponíveis, no contexto das operações de fluxos bidirecionais de transferência de dados entre as aplicações de modelação e de cálculo. O presente relatório analisa as duas perpectivas de investigação: transposição executada com base no formato nativo de dados; transferência com base no formato IFC. O texto descreve as principais fases requeridas no projecto de estruturas, evidenciando a verificação da qualidade dos dados transpostos, através da transferência de dados efectuada segundo os dois procedimentos.

\section{GERAÇÃO DE MODELOS DE ESTRUTURAS}

No contexto BIM, a solução estrutural é concebida, ajustada e rectificada, na forma de um modelo virtual, composto por objectos paramétricos. Neste processo, os utilizadores frequentes de aplicações da Autodesk, admitem facilidade na manipulação do sistema Revit, por apresentar interfaces semelhantes ao AutoCAD. No entanto, a maioria dos fabricantes de aplicações de apoio ao projecto têm seguido a evolução tecnológica requerida no mercado, podendo o modelo paramétrico ser, por exemplo, criado por recurso aos aplicativos AECOsim (Bentley) ou ArchiCAD (Graphisoft). O processo de modelação é aplicado a diversas situações de casos de estudo analisados num contexto académico.

A ferramenta BIM da Autodesk, o Revit, permite a definição do modelo, iniciando com a identificação dos alinhamentos ortogonais e as cotas dos níveis de cada piso. Os objectos paramétricos a utilizar na modelação, são seleccionados a partir da biblioteca de famílias da especialidade de estruturas, disponível no sistema (Sampaio, 2018). Cada objecto, pertencente a famílias de pilares, vigas, paredes de suporte, lajes e sapatas, é seleccionado e adaptado à solução estrutural estabelecida, de acordo com a geometria e com o material a aplicar. As soluções analisadas são em betão armado, e o sistema permite atribuir aos materiais, betão e aço, os valores correspondentes às propriedades mecânicas, nomeadamente, o módulo de elasticidade, o coeficiente de Poisson e o peso volúmico, dos tipos específicos escolhidos (Figura 1).

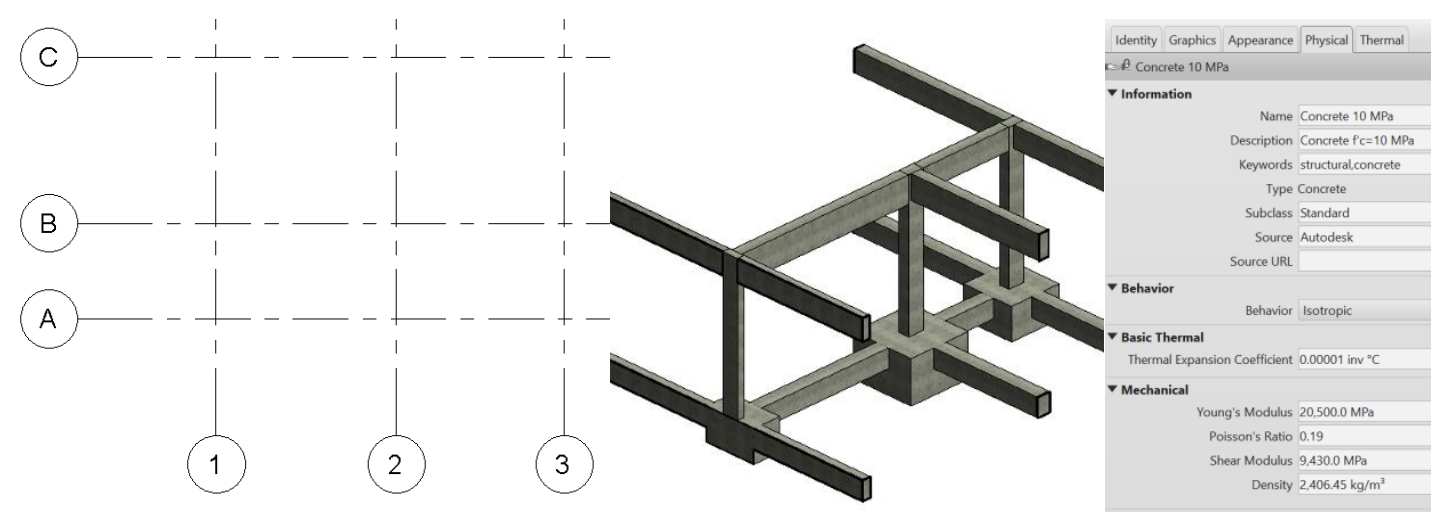

Figura 1. Bases da modelação (alinhamentos, pilares, vigas e sapatas) e propriedades mecânicas 
O edifício localizado em Lisboa, com oito pisos elevados, apresenta uma solução vigada em betão armado com uma distribuição regular dos elementos resistentes (Sampaio et al., 2019). No contexto BIM, a primeira etapa consiste na criação do modelo virtual referente à solução estrutural adotada. Com base na informação fornecida, no formato de desenhos AutoCAD do projecto de estruturas estabelecido, é criado o modelo geométrico, composto por objectos paramétricos. O sistema admite a funcionalidade de discretização dos elementos resistentes, possibilitando a visualização do correspondente modelo de elementos finitos referido como o modelo analítico. Cada elemento é representado pelo seu eixo (vigas e pilares) ou pela sua superfície média (laje ou parede resistente) associado a propriedades requeridas no cálculo (áreas e inércias). $\mathrm{O}$ projectista deve verificar a consistência de todas as ligações previamente à transposição do modelo para o sistema de dimensionamento estrutural (Figura 2).
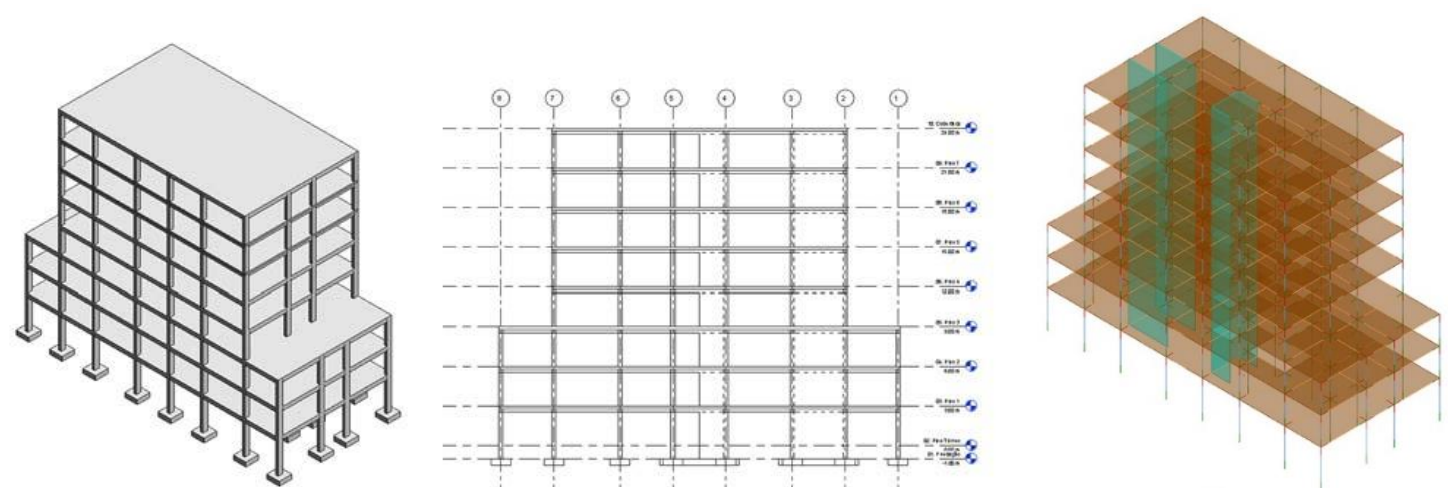

Figura 2. Modelo de estruturas de edifício alto criado no Revit

Uma moradia unifamiliar de piso térreo, localizado em Santarém, foi igualmente modelado por recurso ao sistema Revit. A solução estrutural estabelecida foi elaborada sobre o modelo BIM de arquitectura, permitindo julgar da adequabilidade da estrutura no contexto arquitectónico (Figura 3). A solução de pré-dimensionamento apresenta uma laje maciça fungiforme com vigas periféricas e pilares interiores isolados, com um sistema de fundação composta por sapatas e lintéis no contorno.

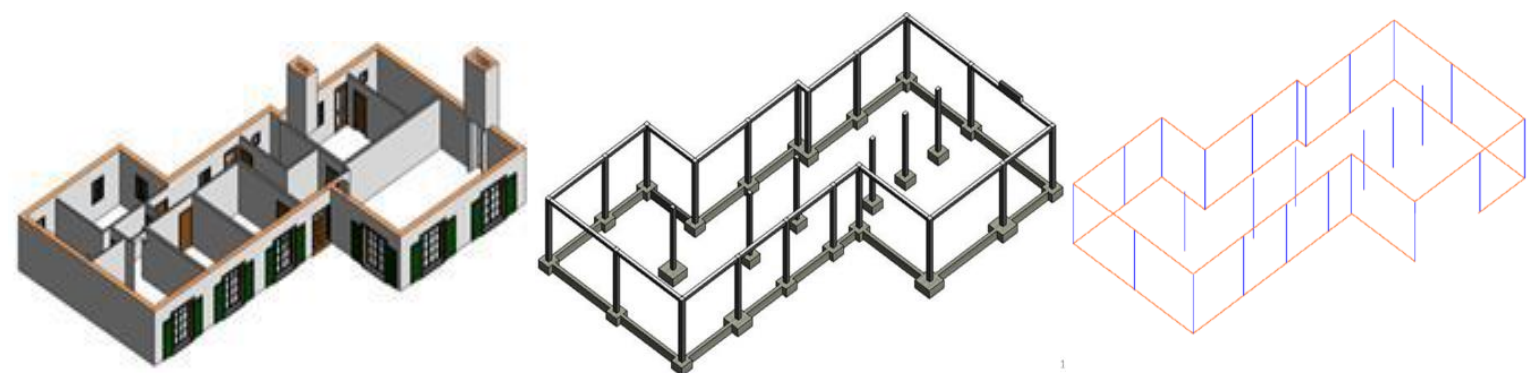

Figura 3. Modelos de arquitectura e de estruturas criados no Revit

Ainda por recurso ao mesmo sistema BIM de modelação, foram elaborados os modelos de arquitectura e de estruturas, referentes a uma moradia de piso térreo e elevado e cobertura inclinada, situada na zona transmontana do Pais (Figura 5) (Azevedo, 2015).
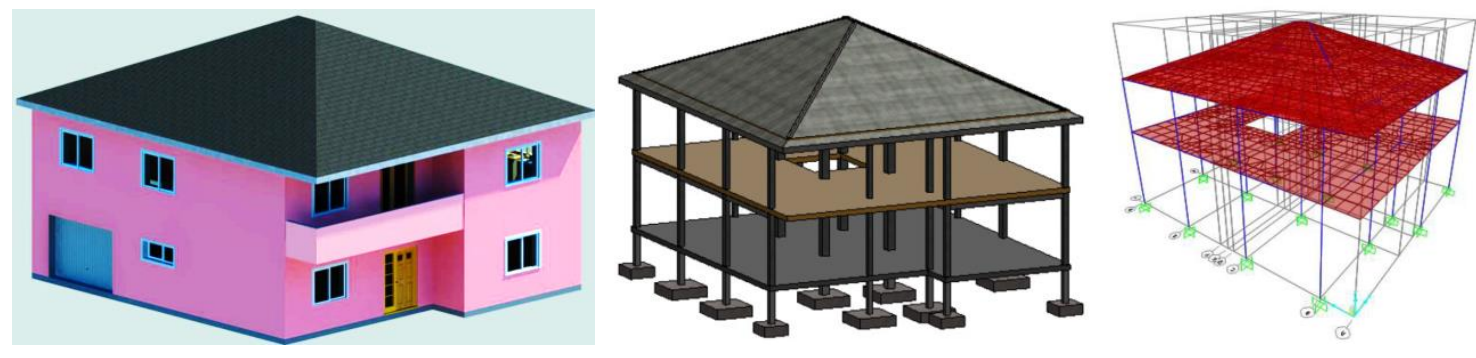

Figura 4. Modelos de arquitectura e de estruturas criados no Revit 
O edifício de ocupação hoteleira situada no Algarve, foi modelada por recurso ao sistema ArchiCAD (Graphisoft), tendo sido criados os modelos dos projectos de arquitectura e de estruturas (Farinha, 2018). A solução estabelecida consiste na disposição de lajes fungiformes, em grande parte do edifício, de modo que as redes dos sistemas hidráulicos e do ar condicionado, pudessem ser posicionadas adequadamente, e para poder conceber a configuração orgânica considerada na arquitectura (Figura 5).
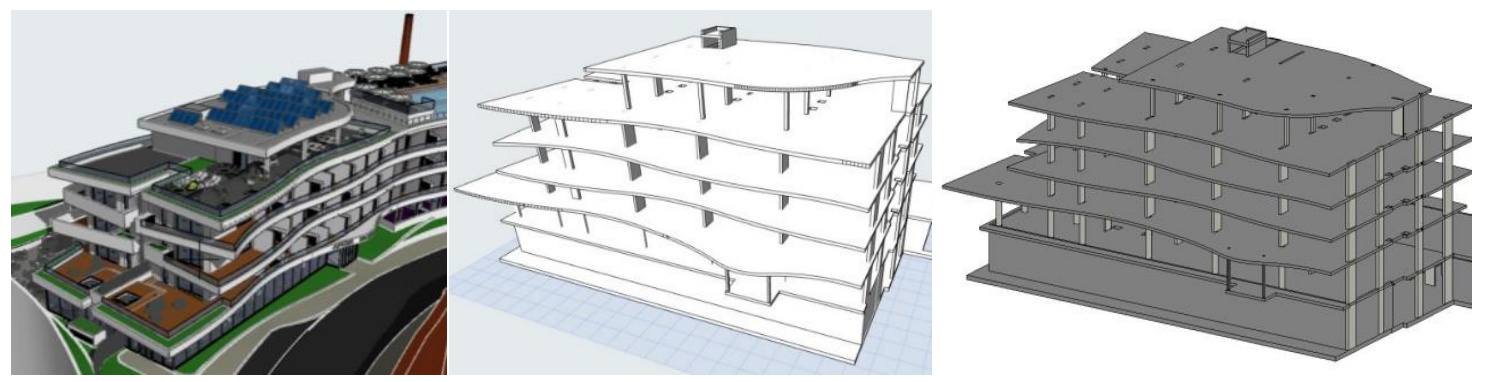

Figura 5. Modelos de arquitectura e de estruturas criados no ArchiCAD

No processo de modelação de um edifício de apoio à área técnica de uma barragem, foi utilizado o sistema AECOsim Building Designer (Bentley) 0. Como base da modelação foram importados os desenhos $d w g$ e por recurso aos elementos paramétricos disponibilizados no sistema, foi criada a estrutura em betão armado (Figura 6). O sistema admite, igualmente, a representação do modelo analítico, discretizando as vigas e os pilares, através de elementos lineares (frames), e as lajes e as paredes por elementos planares (shell).
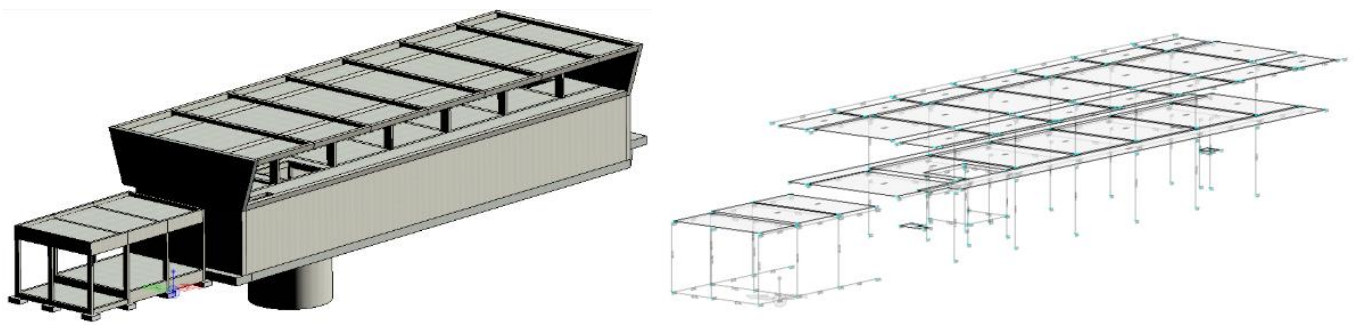

Figura 6. Modelo de estrutura de edifício de apoio a barragem por recurso a AECOsim

Contudo, qualquer um dos sistemas de modelação não admite nenhuma capacidade de cálculo estrutural. Assim, a elaboração do projecto de estrutura, requer que o modelo criado seja transposto para um software de dimensionamento estrutural. Esta operação é acompanhada, em cada um dos casos, com uma especial atenção relativamente ao nível de qualidade da consistência do modelo transposto.

\section{CÁlCUlo eSTRUTURAL}

O modelo de estruturas criado é, então, transposto para o software de cálculo, é verificada a sua consistência geométrica, são aplicadas as combinações de cargas regulamentares e são determinadas as deformações e os esforços, de forma a avaliar a segurança da estrutura em relação aos estados limite último (ELU) e de serviço (ELS). Posteriormente, são produzidos os desenhos de pormenorização de armaduras, por recurso às funcionalidades dos sistemas de cálculo ou directamente nas aplicações de modelação. Os sistemas de cálculo utilizados são o SAP (CSI) (Azevedo, 2015) (Serra, 2015), o Robot (Autodesk) (Sampaio et al., 2019) e o ETABS (CSI) (Farinha, 2018). 


\subsection{Transposição de Modelos}

O formato nativo de dados é utilizado apenas na transferência efectuada entre o Revit e o Robot, pois pertencem à mesma empresa. A transferência de dados nas restantes situações é apoiada no padrão IFC ou nas ligações do tipo add-in, plug-in e extensions, disponibilizadas nos sistemas, permitindo estabelecer algum grau de interoperabilidade. A avaliação do nível da capacidade de interoperabilidade existente é analisada sobre o modelo transposto:

- Transposição Revit/Robot. Os modelos criados no Revit, foram transferidos para o software de cálculo, e analisada a correcção de cada modelo transposto. Foi necessário efectuar algumas correcções, nomeadamente, os elementos de escada não são reconhecidos no sistema, tendo sido modelados como lajes inclinadas, e as sapatas não são transpostas tendo sido consideradas como apoios (Figura 7). Como ambos os sistemas, Robot e Revit pertencem à Autodesk, é verificado um nível de interoperabilidade elevado, embora não totalmente isento de erros (Sampaio et al., 2019).

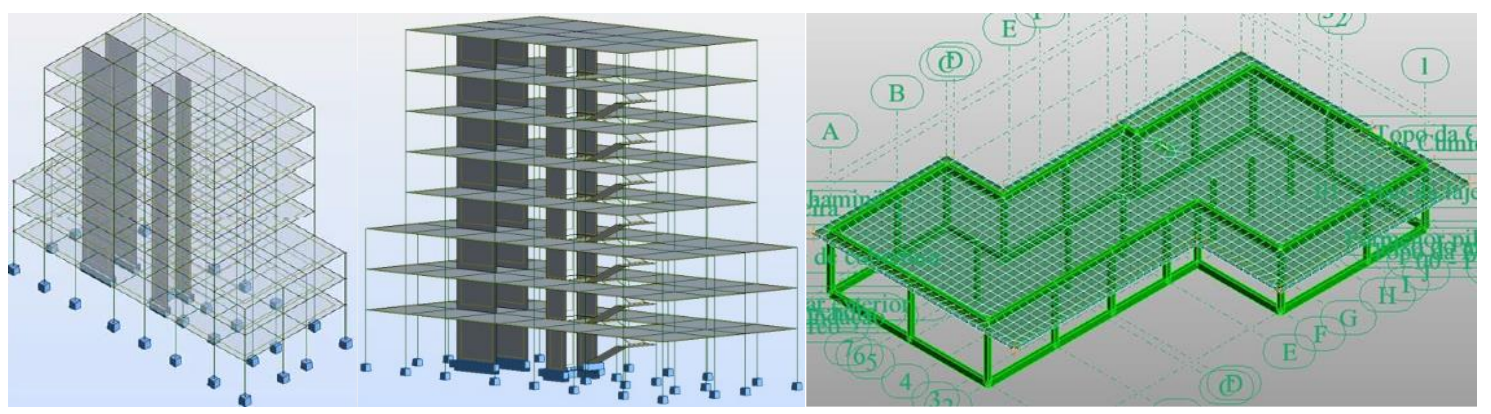

Figura 7. Modelos de estruturas transpostos do Revit para o Robot

- Transposição Revit/SAP. O processo de exportação do modelo para o software de cálculo estrutural foi realizado com base no plug-in, CSIXRevit. A informação transposta considera os elementos estruturais do edifício (sapatas, pilares, vigas e lajes) e, ainda, os alinhamentos. O material, betão C30/37 e aço A500 NR SD, e correspondentes propriedades são correctamente identificados (Figura 8). No entanto, foi necessário efetuar alguns ajustes, nomeadamente, em relação ao eixo analítico de elementos finitos lineares e as ligações rígidas estabelecidas no modelo Revit não são reconhecidas no $S A P$. A modelação efetuada no Revit é bastante intuitiva e as correções requeridas no SAP são mínimas.
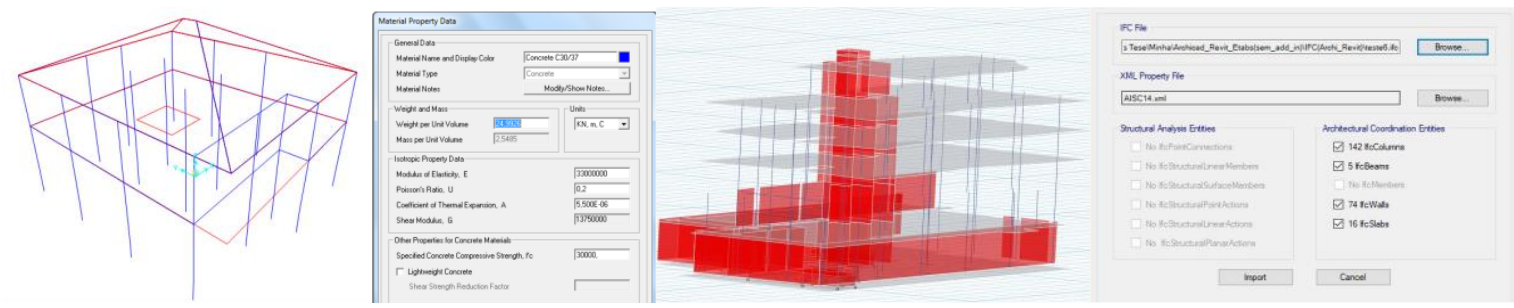

Figura 8. Modelo Revit transposto para o SAP e modelo ArchiCAD para o ETABS

- Transposição ArchiCAD/ETABS. O modelo criado no software ArchiCAD, foi arquivado no formato IFC, e, posteriormente, transposto para o aplicativo ETABS (CSI). Embora, no procedimento tenham sido seleccionados especificamente os tipos IfcColumns, IfcWalls ou IfcSlabs, o resultado de uma análise geométrica efectuada ao modelo transferido, identifica alguns erros de inconsistência, nomeadamente, um elevado número de elementos adjacente desconectados, obrigando a uma verificação e a ajustes pontuais. Este trabalho adicional é demorado, mas é necessário para a obtenção de um modelo de cálculo correcto para a execução do dimensionamento (Figura 8).

- Transposição AECOsim/SAP. O modelo é arquivado no formato IFC e é transferido para o SAP. Este sistema consegue interpretar apenas alguns dos elementos, nomeadamente, ifcStructuralPointConnection, ifcBeam, ifcColumn, ifcSlab, ifcStructuralCurveMember e, ainda, ifcStructuralSurfaceMember, não sendo reconhecidas as sapatas e as escadas. Adicionalmente, são 
correctamente transferidas as secções e espessuras de cada elemento. Contudo, não é transferida nenhuma informação relativamente a condições de fronteira ou a cargas (Figura 9). A funcionalidade do $S A P$, merge joints, permite juntar os nós que distam um valor limite mínimo definido pelo utilizador, mas não verifica a ortogonalidade que os elementos devem apresentar entre si, conduzindo a situações incorretas. A interoperabilidade entre os dois sistemas é, ainda, limitada, no entanto, a possibilidade de exportar o modelo analítico a partir do modelo paramétrico, facilmente gerado no sistema modelador, contribui para a agilização do processo e ao aumento de produtividade (Serra, 2015).

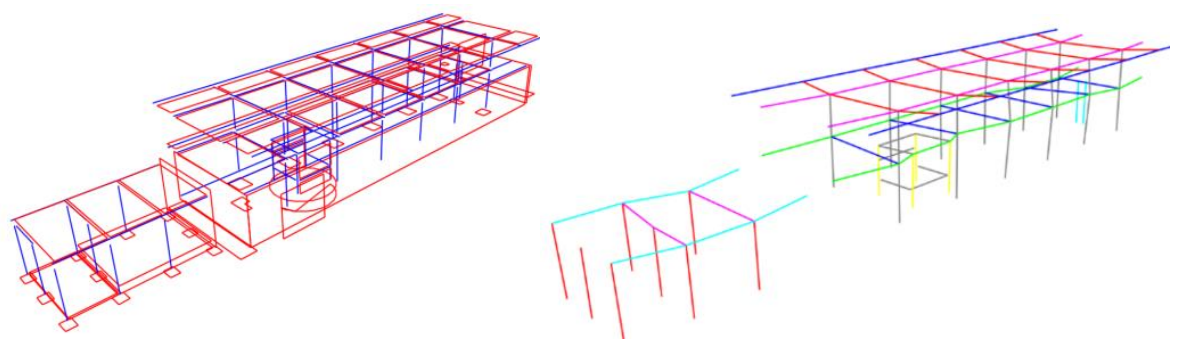

Figura 9. Modelo de estruturas AECOsim transposto para o $S A P$ e retificação com merge joints

\subsection{Verificação de Resistência Estrutural}

Embora o Revit permita a aplicação de cargas e o estabelecimento de combinações para uma posterior análise de segurança aos estados limite último (ELU) e de serviço (ELS), as cargas referentes à acção sísmica não são correctamente transpostas assim como os coeficientes das combinações. Assim todas as cargas e combinações foram aplicadas em cada sistema de cálculo. Sobre o modelo transposto, em cada sistema de cálculo são consideradas as acções regulamentares: as cargas permanentes relativas ao peso próprio estrutural (PP), que é assumida de uma forma automática pelos sistemas de cálculo, com base no peso específico do betão armado; a acção permanente $(\mathrm{CP})$ relativa ao revestimento, distinto por tipo de utilização, e o peso distribuído das paredes interiores; a ação variável (SC) considera o tipo de utilização que é distinto em cada piso ou zona; em relação à acção sísmica, quantificada por espectros de resposta médios definidos regulamentarmente, foi considerado o coeficiente de sismicidade em função da localização de cada edifício.

Para a determinação da deformada da estrutura foram consideradas duas combinações de ações:

$1 \mathrm{PP}+1 \mathrm{CP}$ e $1 \mathrm{PP}+1 \mathrm{CP}+0,4 \mathrm{SC}$.

Para a determinação dos esforços máximos de dimensionamento na estrutura foram consideradas as combinações:

1,35PP+1,35CP+1,5SC, 1PP+1CP+0,4SC+1,5SISM1 e 1PP+1CP+0,4SC+1,5SISM2.

Os resultados são obtidos na forma de diagramas e modelos $3 \mathrm{D}$, de deformações e de esforços, e ainda de notas de cálculo, base da determinação de área de armadura a estabelecer para cada elemento estrutural. Os sistemas de cálculo referidos admitem uma elevada capacidade de automatização de desenhos.

\subsection{Centralização de Dados}

Contudo, de acordo com o conceito base da metodologia BIM, a concepção das diferentes disciplinas deve ser elaborada sobre um modelo único centralizador de toda a informação gerada, para uma posterior consulta ou manipulação. Nesse sentido, o resultado do cálculo deve ser transferido para o modelo BIM de estruturas inicial, actualizando a sua base de dados que deverá ser acessível aos técnicos envolvidos. O processo de transferência inverso apresenta, contudo, um volume bastante superior de incorrecções, razão frequentemente apontada para justificar a resistência da implementação do BIM no projecto de estruturas:

- Transposição Robot/Revit. As armaduras podem ser pormenorizadas no sistema Robot e, posteriormente, ser transpostas para o modelo Revit, ou então podem ser transpostas apenas as áreas necessárias em cada elemento, e a distribuição de varões ser definida no Revit. Em relação a lajes, vigas e pilares, os resultados de cálculo de varões e a armação realizada no Robot, são correctos mas a transposição da informação da pormenorização para a Revit é ainda limitada: no Robot as armaduras as lajes são distribuídas automaticamente, com base nos valores atribuídos pelo utilizador aos parâmetros relativos ao espaçamento 
e aos diâmetros dos varões; as vigas e pilares são armadas de um modo automático, sob a indicação do espaçamento entre cintas ou estribos e do número de barras longitudinais a colocar em cada face, seguindo um processo iterativo. Contudo, quando executada a transposição do Robot para o Revit, são verificadas várias incorrecções: nalguns casos, as armaduras de vigas excedem o seu limite geométrico e os estribos são representados com alguma sobreposição; as armaduras de lajes e das sapatas não são transferidas, sendo, apenas, possível importar os desenhos $d x f / d w g$ da pormenorização efectuada no Robot e que servem de base à elaboração da pormenorização 3D (Figura 10) (Sampaio et al., 2019).

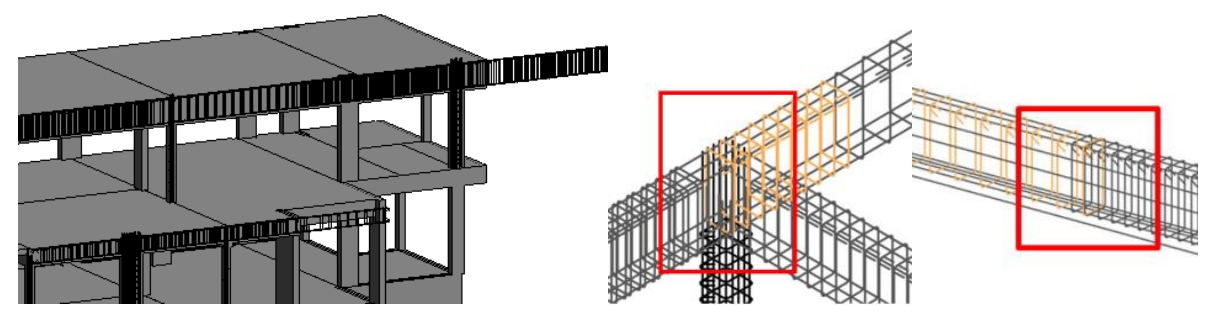

Figura 10. Incorrecções verificadas sobre o modelo transporto

- Para a definição, no Revit, da pormenorização de armaduras a estabelecer sobre cada elemento resistente do modelo transposto, pode ser utilizada a funcionalidade Revit extensions reinforcement, permitindo uma intuitiva e rápida a colocação e anotação dos varões das armaduras nos elementos. Adicionalmente, pode ser usado o complemento AutoCAD Structural Detailing, vinculado ao Revit, permitindo complementar com o detalhe usualmente exigido na documentação gráfica do projecto (Figura 11). Um dos aspectos da metodologia BIM é a capacidade de extração de diverso tipo de informação do modelo. Utilizando a capacidade do Revit, forma obtidas folhas de desenho, organizadas de acordo com a forma usual de apresentação da documentação grafica do projeto, e de distinto tipo de tabelas referentes a quantidades do material, betão e aço, listadas por elementos, pisos, volumes ou diâmetros de varões e de areas de cofragem. As tabelas de quantidades podem ser usadas no apoio à orçamentação do projeto e os mapas de aramduras no estaleiro da obra ou na pré-fabricação.

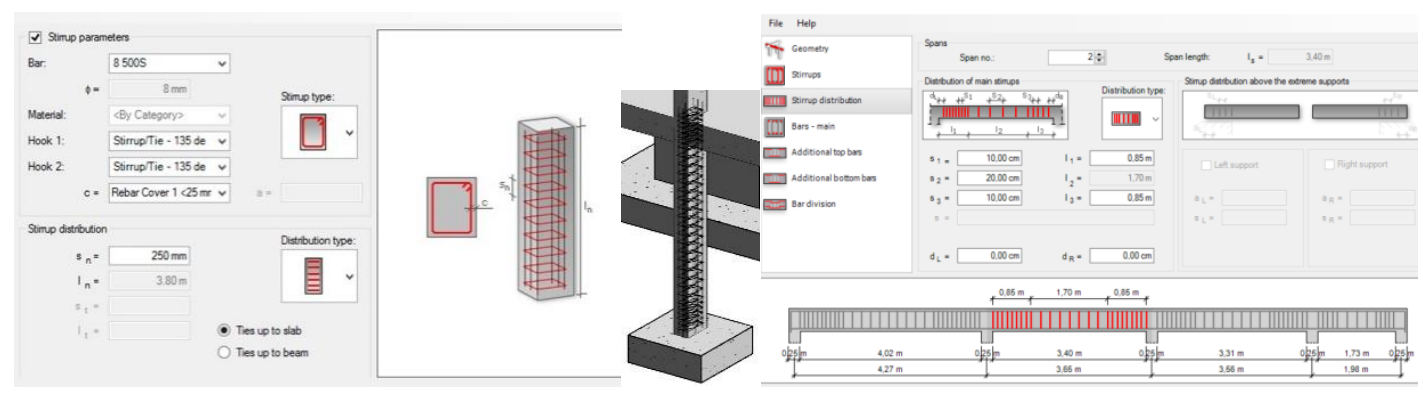

Figura 11. Interfaces da funcionalidade Revit extensions reinforcement

- Transposição SAP/Revit. A distribuição de armaduras efectuada no SAP não é passível de ser transmitida para o Revit, devendo os desenhos produzidos no $S A P$ ser transpostos, no formato $d w g$ ou $d x f$, e proceder à armação de cada elemento no Revit.

- Transposição ETABS/ArchiCAD. Embora o ETABS possua uma elevada capacidade de produção de peças desenhadas, com a pormenorização de armaduras parametrizada com o cálculo, esta informação não é passível de ser exportada no formato IFC, e não admite nenhum add-in ou plug-in de suporte à transposição. Assim, o processo de pormenorização das armaduras foi, posteriormente, executado no ArchiCAD, com base no resultado do dimensionamento transposto. Perante as evidentes limitações, o trabalho académico foi realizado com o recurso a um sistema intermédio, o Revit, tendo sido estudado em pormenor os fluxos de transferência ArchiCAD/Revit/ETABS e ETABS/Revit/ETABS, com conclusões bastante positivas (Farinha, 2018). 
- Transposição SAP/AECOsim. O processo de exportação do modelo SAP, apenas com o resultado do cálculo, para o AECOsim é efectuado no formato IFC. O modelo é transposto automaticamente, resultando o modelo com a omissão dos elementos de parede resistente e sem a informação relativa a cargas e condições de fronteira (Serra, 2015).

\section{CONCLUSÕES}

A estudo revela o nível de interoperabilidade existente entre os sistemas de modelação e de cálculo de base BIM, mais frequentemente utilizados, na elaboração do projecto de estruturas. Como resultados principais podem ser referidos os seguintes aspectos: Há vantagens no uso de plataformas integradas Revit/Robot, pois o recurso ao formato IFC apresenta alguma incorrecção, no contexto das estruturas; $\mathrm{O}$ fluxo de dados no sentido modelação/cálculo pode ser efectuado com bastante confiança, enquanto que o fluxo de dados no sentido inverso é ainda pouco eficiente; É adequado realizar a pormenorização de armaduras no sistema de cálculo, pois admite uma elevada capacidade para a produção de desenhos e modelos 3D e, posteriormente, se as armaduras transpostas apresentarem alguma incorrecção, são facilmente ajustadas; Quando não é possível transferir a informação 3D das armaduras (lajes, escadas e sapatas) é permitido importar os desenhos de pormenorização, e proceder à sua redefinição no sistema modelador, por recurso a capacidades do tipo add-in, plug-in ou extensions. De um modo progressivo e com evidentes resultados positivos, a implementação do BIM tem vindo a ser introduzida em todas a actividades inerentes ao sector da Construção. O presente texto sintetiza os principais aspectos relacionados com a análise de interoperabilidade referente aos sistemas de base BIM, de uso mais frequente, utilizados no âmbito do projecto de estruturas, sendo apresentadas as limitações encontradas, referidas as estratégias de solução e identificados os benefícios. As vantagens estão, essencialmente, relacionadas com a fácil modelação inicial, com alguma capacidade de transferência de informação pós-cálculo e, ainda, com a potencialidade das ferramentas BIM de obter tabelas de quantidades de material.

\section{REFERÊNCIAS}

Azevedo, V.S., 2015, Análise do modelo BIM numa perspectiva do projecto de estruturas, Dissertação de Mestrado Integrado em Engenharia Civil, IST, Lisboa, Portugal.

BuildingSMART, 2019, Industry Foundation Classes (IFC) - buildingSMART Technical, https://technical.buildingsmart.org/standards/ifc/.

Farinha, T.S., 2018, Análise do processo de transposição de informação entre modelos de estrutura em ambiente BIM, Dissertação de Mestrado Integrado em Engenharia Civil, IST, Lisboa, Portugal.

NIBS News \& Press (2013), BIM educational strategy the focus of National Institute of Building Sciences Conference. Building Innovation, http://www.nibs.org/news/113631/.

Sacks, R., Eastman, C.M., Teicholz, P., Lee, G., 2018, BIM handbook: A guide to Building Information Modeling for owners, designers, engineers, contractors, and facility managers, $3^{\text {rd }}$ ed., ISBN: 978-1-119-28755, 2018, 688 pgs Copyright (C) 2018 by John Wiley \& Sons, Inc.

Sampaio, A.Z., 2017, BIM as a Computer-Aided Design methodology in Civil Engineering, JSEA - The Journal of Software Engineering and Applications, ISSN: 1945-3124 (online), $\mathrm{n}^{\circ}$ 10, pp. 194-210, https://doi.org/10.4236/jsea.2017.102012.

Sampaio, A.Z., 2018, Introdução ao BIM: Geração do modelo de estruturas, Apontamentos didacticos de Desenho Assistido por Computador, Mestrado Integrado em Engenharia Civil, IST, Lisboa.

Sampaio, A.Z., Novais, J.N., Diniz, J.P., 2019, Analysis of BIM implementation in structural projects, CMN 2019 Congresso de Métodos Numéricos em Engenharia, Guimarães, Portugal.

Serra, P.M., 2015, Análise da implementação de processos BIM aplicados ao projeto de estruturas, Dissertação de Mestrado Integrado em Engenharia Civil, IST, Lisboa, Portugal. 